\title{
Mass and density of asteroids (4) Vesta and (11) Parthenope
}

\author{
B. Viateau and M. Rapaport \\ Observatoire de Bordeaux, UMR 5804, CNRS, BP 89, 33270 Floirac, France
}

Received 29 August 2000 / Accepted 7 February 2001

\begin{abstract}
As mentioned in a previous paper (Viateau \& Rapaport 1997), the orbit of asteroid (17) Thetis is strongly perturbed by two large minor planets, (4) Vesta and (11) Parthenope. These strong gravitational perturbations enabled us to determine both the mass of Vesta and the mass of Parthenope. We also independently derived the mass of Vesta from observations of (197) Arete. The weighted mean of the two results gives the value $(1.306 \pm 0.016) 10^{-10} M_{\odot}$ (solar mass) for the mass of Vesta, and its derived mean density is $(3.3 \pm 0.5) \mathrm{g} / \mathrm{cm}^{3}$. For (11) Parthenope, the values $(2.56 \pm 0.07) 10^{-12} M_{\odot}$ and $(2.3 \pm 0.2) \mathrm{g} / \mathrm{cm}^{3}$ were obtained, respectively for its mass and its mean density.
\end{abstract}

Key words. minor planets, asteroids - astrometry - ephemerides

\section{Introduction}

Large asteroids induce significant gravitational perturbations on the orbits of other solar system bodies. The uncertainties on their mass are a problem for the calculation of accurate planetary ephemerides (Standish et al. 1995; Hilton et al. 1996). As an example, these uncertainties cause problems in improving the determination of the relativistic precession of the perihelion of Mars' orbit, because of long-period resonances between the planet and some large asteroids (Eubanks 1996). (4) Vesta is one of the strongest perturbers of Mars' orbit and one of those causing the largest uncertainties on its ephemeris. Vesta also induces strong gravitational perturbations on the orbits of many other asteroids. For example, Bange (1998) showed that the mass of asteroid (20) Massalia obtained from perturbations on (44) Nysa was strongly correlated with the mass of Vesta. Thus, a very accurate value of the mass of Vesta is needed.

The mass of a large asteroid can directly be determined from its gravitational perturbations on other bodies, named "target" bodies. Most of the time, the target bodies are other asteroids. In a previous paper (Viateau \& Rapaport 1997), hereafter referred to as Paper I, we pointed out that recent very close encounters occurred between asteroid (17) Thetis and two larger minor planets, (4) Vesta and (11) Parthenope, respectively in 1996 and 1997. In that paper, a first value of the mass of Parthenope was obtained from the analysis of the observations of Thetis, thanks to a previous very close encounter in 1968. We also pointed out that, due to the very strong

Send offprint requests to: M. Rapaport, e-mail: rapaport@observ.u-bordeaux.fr perturbation of Vesta on the orbit of Thetis, only a few years of additional ground-based observations of the latter asteroid would be sufficient to obtain a very precise determination of the mass of Vesta, which is a rare phenomenon in asteroid mass determination.

This determination of the mass of Vesta from observations of Thetis is now possible. In order to improve the accuracy of the determination of this mass, we also used the observations of a second target asteroid, (197) Arete. Arete was the first asteroid used for the determination of the mass of Vesta, by Hertz (1966). A few other determinations of this mass have been made since, most of them from perturbations on Arete (Table 1), which now appears to be the second-best target asteroid after Thetis for the determination of the mass of Vesta. The final result presented in this paper is obtained from the resolution of the equations associated with the observations of Thetis and Arete. These equations are treated as a single system, and solved by the Gauss-Seidel iterative method, so that the masses of Vesta and Parthenope are determined together, with their correlation coefficient.

\section{Description of the close encounters}

\section{1. (4) Vesta-(17) Thetis}

The evolution with time of the distance between Thetis and Vesta is shown in Fig. 1a. It can be seen that both asteroids come close to each other every 55 years. The last encounter in 1996 was unusual, because both asteroids had almost the same osculating elements and consequently the distance between the asteroids remained smaller than 0.4 AU from March 1995 to June 1998 (Fig. 1b). 
Table 1. Status of the mass determination of Vesta, before the work presented here

\begin{tabular}{lcll}
\hline Author & $\begin{array}{c}\text { perturbed } \\
\text { bodies }\end{array}$ & $\begin{array}{c}\text { mass } \\
\left(10^{-10} M_{\odot}\right)\end{array}$ \\
\hline Hertz (1966) & $(197)$ Arete & 1.17 & 0.10 \\
Hertz (1968) & $(197)$ Arete & 1.2 & 0.08 \\
Schubart \& Matson (1979) & $(197)$ Arete & 1.38 & 0.12 \\
Standish \& Hellings (1989) & Mars & 1.5 & 0.3 \\
Goffin (1991) & (197) Arete & 1.33 & \\
Sitarski (1995) & $(197)$ \& (486) & 1.396 & 0.043 \\
Standish et al. (1995) & DE403 solution & 1.34 & \\
Standish et al. (1998) & DE405 solution & 1.3 & \\
Hilton (1999) & (1) \& (2) & 1.69 & 0.11 \\
Michalak (2000) & 25 asteroids & 1.36 & 0.05 \\
\hline
\end{tabular}

(a)

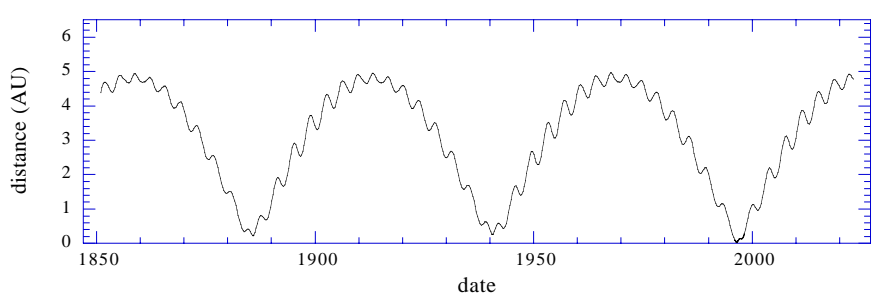

(b)

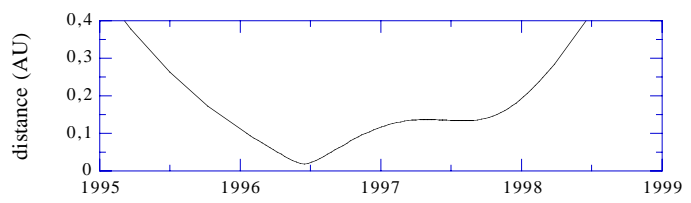

Fig. 1. Distance between (17) Thetis and (4) Vesta. a) From 1850 to 2023 . b) From 1995 to 1999

The minimum was 0.0194 AU in June 1996, with a relative velocity of $1.2 \mathrm{~km} \mathrm{~s}^{-1}$. A much smaller relative velocity of $0.2 \mathrm{~km} \mathrm{~s}^{-1}$ was reached in March 1997. Since Vesta is the second most massive asteroid of the main belt, with a mass equal to about $30 \%$ of the mass of Ceres (Table 2), such an encounter induced a very strong gravitational perturbation on the orbit of Thetis, up to $1^{\prime \prime}$ per year on average (Fig. 2). Since the best ground-based asteroid observations currently have an accuracy better than $0.1^{\prime \prime}$, a few years of such data are sufficient to obtain the value of the mass of Vesta with a very small standard deviation.

Reciprocally, Thetis, with an assumed mass of $5 \times$ $10^{-13} M_{\odot}$ (its diameter measured by the IRAS satellite is $93 \mathrm{~km}$ and its mean density was assumed to be $2.4 \mathrm{~g} / \mathrm{cm}^{3}$ ), is almost 300 times less massive than Vesta, and its perturbation on the orbit of Vesta is always less than $0.2^{\prime \prime}$ in right ascension, too small for its mass to be determined.

\section{2. (11) Parthenope-(17) Thetis}

Since its discovery in 1852, Thetis has experienced two close encounters with (11) Parthenope (Fig. 3). The first one, in February 1968, was extremely close, with a minimal distance of $0.0016 \mathrm{AU}$ (i.e. about $240000 \mathrm{~km}$ ) and

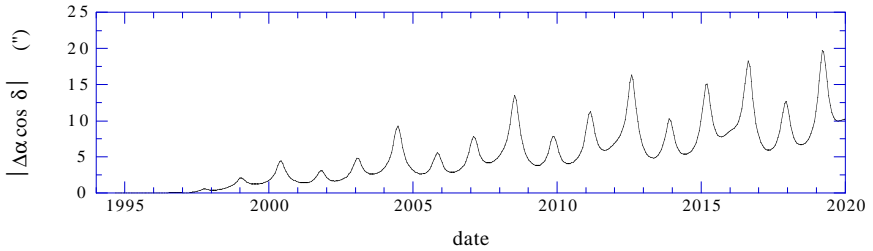

Fig. 2. Effect of the gravitational perturbation of (4) Vesta on the orbit of (17) Thetis in right ascension, starting forward numerical integration at epoch JD $2450000.5=1995$ Oct. $10.0 \mathrm{TT}$ and assuming a mass of $1.3510^{-10} M_{\odot}$ for Vesta

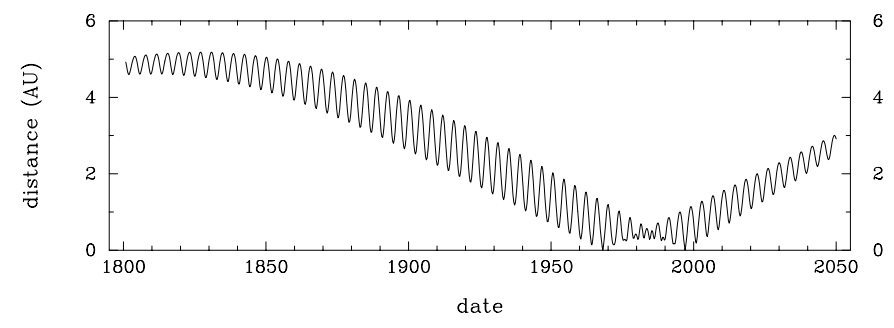

Fig. 3. Distance between (11) Parthenope and (17) Thetis from 1800 to 2050

a relative velocity of $2.3 \mathrm{~km} \mathrm{~s}^{-1}$. This is this encounter that allows the mass of Parthenope to be determined, with a gravitational perturbation up to $15^{\prime \prime}$ in right ascension (Fig. 4). The second encounter took place in January 1997 with a minimal distance of 0.0054 AU and a relative velocity of $2.4 \mathrm{~km} \mathrm{~s}^{-1}$. This recent encounter was not yet effective when the first determination of the mass of Parthenope was made (Paper I), and allows now suppress a more precise determination of this mass.

$0.0016 \mathrm{AU}$ is the smallest minimal distance between two asteroids we had to deal with in asteroid mass determination. Moreover, both asteroids are not very different in size: the IRAS diameter of Parthenope is $162 \mathrm{~km}$, and the diameter of Thetis is $93 \mathrm{~km}$. The closer two asteroids come to each other, the more accurately their mutual distance must be calculated. The necessity of taking into account the reciprocal perturbation of Thetis when the orbit of Parthenope was computed by numerical integration was considered. However, the maximal deviation of the orbit of Parthenope caused by Thetis was about $2^{\prime \prime}$, which caused a too small relative change on the mutual distance for the determination of the mass of Parthenope to be affected. We therefore neglected this effect. The deviation of $2^{\prime \prime}$ of the orbit of Parthenope was also too small for the mass of Thetis to be determined.

\section{3. (4) Vesta-(197) Arete}

Vesta and Arete come close to each other every 18 years (Fig. 5). Among the 7 close encounters experienced by the two asteroids in the period 1879-1999, the first one in May 1885 was the closest, with a minimal distance of $0.018 \mathrm{AU}$ and a relative velocity of $2.2 \mathrm{~km} \mathrm{~s}^{-1}$. Since that date, the minimal distance tends to increase at each encounter. The last encounter between the two asteroids 


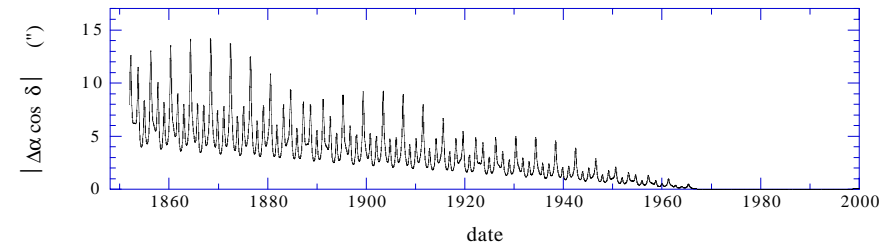

Fig. 4. Effect of the gravitational perturbation of (11) Parthenope on the orbit of (17) Thetis in right ascension, backward numerical integration at epoch JED $2450000.5=$ 1995 Oct. $10.0 \mathrm{TT}$ and assuming a mass of $2.610^{-12} M_{\odot}$ for Parthenope

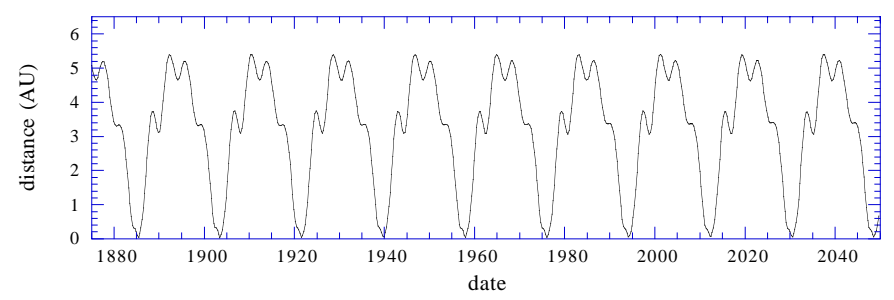

Fig. 5. Distance between (4) Vesta and (197) Arete from 1875 to 2050

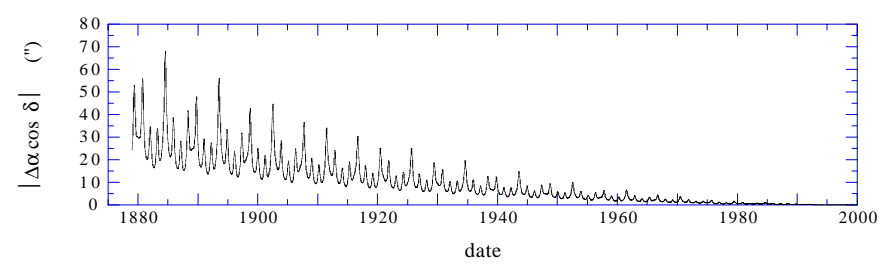

Fig. 6. Effect of the gravitational perturbation of (4) Vesta on the orbit of (197) Arete in right ascension, starting backward numerical integration at epoch JED $2450000.5=1995$ Oct. $10.0 \mathrm{TT}$ and assuming a mass of $1.3510^{-10} M_{\odot}$ for Vesta

occurred in March 1994 with a minimal distance of 0.042 AU. This series of encounters induced a strong perturbation on the orbit of Arete, up to $60^{\prime \prime}$ in 100 years, which allows the mass of Vesta to be obtained with good accuracy. On the other hand, the determination of the mass of Arete from this encounter is not possible, because its gravitational pertubation on the orbit of Vesta is too small (the IRAS diameter of Arete is only $32 \mathrm{~km}$ ).

\section{Method}

\subsection{System resolution}

The unknowns to be determined are the masses of Vesta and Parthenope, and the orbital elements of Thetis and Arete, from astrometrical observations of the two latter asteroids. The equation matrix $M$ has the following structure:

$M=\left(\begin{array}{ccc}A_{1} & 0 & B_{1} \\ 0 & A_{2} & B_{2}\end{array}\right)$

where $A_{1}$ and $A_{2}$ are 6-columns blocks and correspond to the orbital elements of Thetis and Arete, respectively. Blocks $B_{1}$ and $B_{2}$ have 2 columns, each of these columns respectively corresponds to the masses of Vesta and Parthenope. Calculation of singular values of $M$ shows that the problem is well conditioned, the condition number being equal to 440 .

The equation system is solved by using the GaussSeidel iterative algorithm. In this algorithm, the global system is partitioned in blocks and solved by an iterative process, each block being solved independently. The interest of this method is that it converges to the solution of the global system, whatever the starting point, if the matrix of this system is positive definite (e.g. Ralston \& Rabinowitz 1978; Jefferys 1963). This condition is satisfied in our case because the normal equation matrix $M^{\mathrm{t}} M$ is symmetric and regular. That result allows us to choose as initial values for masses and orbits estimates from previous works, which are expected to be close to the solution, so that convergence can be accelerated.

In our case, the chosen initial values were the mass of Parthenope determined in Paper I, $2.5810^{-12} M_{\odot}$, the corresponding orbital data of Thetis, a value of the mass of Vesta estimated from previous works by other authors, $1.3510^{-10} M_{\odot}$, and orbital elements of Arete taken in the "Ephemerides of Minor Planets for 1995" (Batrakov 1994).

The small blocks to be separately solved here are the classical systems including one mass and six orbital elements. In the first step, the mass of Vesta was separately determined from observations of Thetis and then from observations of Arete, each time with the orbital elements of the test asteroid, and a weighting mean of the two values of the mass was calculated. Gravitational perturbations on the orbits of Thetis and Arete from other asteroids including Parthenope were taken into account, as will be detailed in Sect. 3.2.

In the next step, the mass of Parthenope was determined from observations of Thetis. Results from the previous step were used, in particular new orbital elements of Thetis and the new value of the mass of Vesta to take into account its gravitational perturbation on the orbit of Thetis. The global process was iterated and convergence was reached in two iterations.

The following sections describe the orbit computation and data selection procedures used for each mass determination.

\subsection{Orbit computation}

The procedures presented in this section and in the next one are similar to those used in Paper I. The theoretical positions of asteroids (17) Thetis and (197) Arete were calculated for a given date with the Bulirsh and Stoer numerical integration method (Bulirsh \& Stoer 1966). The osculating elements used as initial conditions for Thetis were the ones obtained in Paper I, and expressed for the initial date JD 2450000.5 TT. The orbital elements of Arete were taken in the "Ephemerides of Minor Planets for 1995" (Batrakov 1994, initial date JD = 2450000.5 TT). As discussed in Sect. 3.1, in the case of (17) Thetis, only 
Table 2. Perturbing asteroids used with their mass, and their maximal perturbation in $\alpha \cos \delta$ on orbits of (17) Thetis and (197) Arete for the period covered by the observations, when starting numerical integration on date JD $2450000.5=$ 1995 Oct. 10.0 TT. For the perturbation of Vesta on Thetis, the cases "B" (backward numerical integration) and "F" (forward numerical integration) have been distinguished since the close encounter that enables the mass determination is posterior to the initial date (case "F"). For all other cases, the maximum effect was obtained with backward numerical integration, for dates close to the oldest observations of Thetis and Arete. The values given for the masses of Vesta and Parthenope are the initial values used for the Gauss-Seidel iterative process as described in Sect. 3.1

\begin{tabular}{lccc}
\hline Asteroid & $\begin{array}{c}\text { Mass } \\
\left(10^{-10} M_{\odot}\right)\end{array}$ & \multicolumn{2}{c}{ Max. effect $\left({ }^{\prime \prime}\right)$} \\
& 4.76 & 0.73 & $(197)$ \\
\hline (1) Ceres & 1.2 & 0.63 & 0.31 \\
(2) Pallas & 1.35 & $2.98 \mathrm{~B}$ & 55.5 \\
(4) Vesta & & $2.15 \mathrm{~F}$ & \\
& & 0.04 & 0.28 \\
(10) Hygiea & 0.47 & 14.6 & 0.04 \\
(11) Parthenope & 0.0258 & 0.78 & 0.31 \\
(52) Europa & 0.14 & 0.07 & 0.09 \\
(511) Davida & 0.18 & 0.15 & 0.09 \\
(704) Interamnia & 0.35 & & \\
\hline
\end{tabular}

one mass (Vesta or Parthenope) was determined at a time. The integration of the motion of Thetis and Arete took into account, as a standard procedure, the gravitational perturbations of the nine major planets but Pluto (VSOP82 theory, Bretagnon 1982), the perturbations of Vesta and Parthenope and, in addition, the perturbations of 6 other massive asteroids. These asteroids are listed in Table 2 with the assumed value for their mass, and the resulting maximal perturbation on the orbits of Thetis and Arete. The values of the masses were taken from the literature when possible, or, if not, were deduced from the mean diameter of the asteroids and an estimation of their mean density. For (1) Ceres, the assumed initial value of $4.7610^{-10} M_{\odot}$ (solar mass) is the value obtained by Viateau \& Rapaport (1998). For (2) Pallas, (4) Vesta and (704) Interamnia, the values assumed are close to the mean of all mass determinations for these asteroids. The value used for (10) Hygiea is the result found by Scholl et al. (1987). For (11) Parthenope, the value used was the result found in Paper I. For (52) Europa and (511) Davida, which are respectively CF and C-class asteroids, and for which no direct mass determination has ever been made, the mass was calculated from the diameter of those asteroids given by IRAS, and an assumed mean density of $1.8 \mathrm{~g} / \mathrm{cm}^{3}$.

\subsection{Data selection}

Most of the data used in this work were provided by the Minor Planet Center (USA), through their Extended Computer Service. The MPC data used contained 1170 observations of (17) Thetis, covering the period 18521999, and 396 observations of (197) Arete, for the period 1879-1999. Tables 3 and 4 give the characteristics of these data. About $10 \%$ of the observations of Thetis are photoelectric observations made with the Bordeaux meridian circle from 1984 to 1994, which are presented in detail in Paper I. 156 new observations of Thetis were made between 1996 and 1999, since Paper I was written. 29 of them were made with the Bordeaux and the São Paulo CCD meridian circles as part of a collaboration between Bordeaux Observatory and Instituto Astronomico e Geofisico of São Paulo, Brazil (Viateau et al. 1999). As a standard procedure, all the data were expressed in the ICRF (International Celestial Reference Frame). Since all the observations do not have the same accuracy, a procedure of data selection and weighting, similar to the one described in Paper I, was applied.

First, all the observations provided with a precision worse than $0.1 \mathrm{~s}$ of time in right ascension and 1 arcsec in declination were eliminated. Then, an iterative procedure was used. At the first iteration, the residuals of the observations were calculated using the initial conditions mentioned in Sect. 3.2. Since there were observations of very different epochs and, thus, of different accuracies among the data used, these data were separated into several groups with respect to the epoch of the observations. Each group was made up of observations showing residuals of about the same visual dispersion.

At every iteration, the standard deviation $\sigma$ of the residuals was calculated for each group, and the observations giving residuals over $2.5 \sigma$ were eliminated. Weights corresponding to $\sigma$ were applied to the observations. Corrections for the mass of the perturbing asteroid and the six osculating elements of the perturbed body were calculated by a classical least-squares method, and gave a new solution that was used as initial conditions for the next iteration. In the case of photographic or CCD observations, since right ascension and declination are not independent of each other in the reduction procedure, both coordinates were rejected if one of them gives a residual over $2.5 \sigma$. Iterations were made until convergence.

\section{Results}

\subsection{Mass of Parthenope}

The new value found for the mass of Parthenope is $(2.56 \pm$ 0.06) $10^{-12} M_{\odot}$, and is very close to the value $(2.58 \pm$ 0.10) $10^{-12} M_{\odot}$ obtained in Paper I. The main difference between the two results is the smaller standard deviation on the value of the mass, due to the effect of the second close encounter between Thetis and Parthenope in 1997, as explained in Sect. 2.2. Assuming the mean diameter of Parthenope to be the IRAS value $(162 \pm 3 \mathrm{~km})$ gives a mean density of $2.3 \pm 0.2 \mathrm{~g} / \mathrm{cm}^{3}$ for this S-class asteroid (see Fig. 7 for comparison with other asteroids). 
Table 3. Characteristics of each group of observations of (17) Thetis; "initial number" is the number of observations before elimination by the iterative procedure (but after elimination of the data published with poor precision); "final number" is the number of observations selected; "\% elim" is the percentage of eliminated observations; " $\sigma$ " is the standard deviation of the residuals after the last iteration and "weight" is the final weight applied; unit weight corresponds to a mean precision of 0.5 "

\begin{tabular}{|c|c|c|c|c|c|c|c|}
\hline \multicolumn{2}{|c|}{ observations } & number & $\begin{array}{c}\text { initial } \\
\text { number }\end{array}$ & final & $\%$ elim & $\sigma\left(^{\prime \prime}\right)$ & weight \\
\hline \multirow{2}{*}{\multicolumn{2}{|c|}{$1852-1872$}} & $\alpha$ & 74 & 28 & 62.2 & 3.98 & 0.02 \\
\hline & & $\delta$ & 74 & 28 & 62.2 & 1.57 & 0.1 \\
\hline \multirow{2}{*}{\multicolumn{2}{|c|}{$1879-1957$}} & $\alpha$ & 292 & 206 & 29.5 & 1.17 & 0.2 \\
\hline & & $\delta$ & 292 & 211 & 27.7 & 0.85 & 0.3 \\
\hline \multirow{2}{*}{\multicolumn{2}{|c|}{$1959-1983$}} & $\alpha$ & 231 & 191 & 17.3 & 0.88 & 0.3 \\
\hline & & $\delta$ & 231 & 191 & 17.3 & 0.94 & 0.3 \\
\hline \multirow{2}{*}{\multicolumn{2}{|c|}{ 1984-1994 (global) }} & $\alpha$ & 336 & 278 & 17.3 & 0.24 & \\
\hline & & $\delta$ & 336 & 281 & 16.4 & 0.27 & \\
\hline \multirow[t]{6}{*}{ among which: } & Bordeaux & $\alpha$ & 111 & 108 & 2.7 & 0.15 & 10 \\
\hline & meridian & $\delta$ & 111 & 108 & 2.7 & 0.23 & 4.5 \\
\hline & La Palma & $\alpha$ & 136 & 130 & 4.4 & 0.26 & 3.7 \\
\hline & meridian & $\delta$ & 136 & 133 & 2.2 & 0.27 & 3.5 \\
\hline & Other & $\alpha$ & 89 & 40 & 55.1 & 0.30 & 2.7 \\
\hline & & $\delta$ & 89 & 40 & 55.1 & 0.33 & 2.2 \\
\hline \multirow{10}{*}{ among which: } & global) & $\alpha$ & 156 & 94 & 39.7 & 0.07 & \\
\hline & & $\delta$ & 156 & 97 & 37.8 & 0.07 & \\
\hline & Bordeaux & $\bar{\alpha}$ & 19 & 18 & 5.3 & 0.05 & 119 \\
\hline & meridian & $\delta$ & 19 & 18 & 5.3 & 0.09 & 34 \\
\hline & Valinhos & $\bar{\alpha}$ & 10 & 8 & 20.0 & 0.05 & 88 \\
\hline & meridian & $\delta$ & 10 & 8 & 20.0 & 0.05 & 85 \\
\hline & La Palma & $\alpha$ & 11 & 6 & 45.5 & 0.09 & 33 \\
\hline & meridian & $\delta$ & 11 & 9 & 18.2 & 0.08 & 36 \\
\hline & Other & $\alpha$ & 116 & 62 & 46.6 & 0.08 & 38 \\
\hline & & $\delta$ & 116 & 62 & 46.6 & 0.06 & 62 \\
\hline
\end{tabular}

Table 4. Characteristics of each group of observations of (197) Arete; for details see Table 3

\begin{tabular}{ccccccc}
\hline observations & initial & final & \% elim & $\sigma\left(^{\prime \prime}\right)$ & weight \\
& $\alpha$ & 63 & 48 & 23.8 & 1.35 & 0.1 \\
\hline $1879-1962$ & $\alpha$ & 63 & 49 & 22.2 & 1.36 & 0.1 \\
\hline \multirow{2}{*}{$1966-1993$} & $\alpha$ & 206 & 181 & 12.1 & 0.94 & 0.3 \\
& $\delta$ & 206 & 181 & 12.1 & 1.40 & 0.1 \\
\hline \multirow{2}{*}{$1996-1999$} & $\alpha$ & 90 & 72 & 20.0 & 0.26 & 3.8 \\
& $\delta$ & 90 & 72 & 20.0 & 0.18 & 7.5 \\
\hline
\end{tabular}

\subsection{Mass of Vesta and correlation coefficient}

After convergence, the value of the mass of Vesta obtained from perturbations on Thetis was (1.314 \pm 0.018) $10^{-10} M_{\odot}$, and the result obtained from perturbations on Arete was $(1.294 \pm 0.023) 10^{-10} M_{\odot}$. Tables 5 and 6 present the corresponding new orbital elements obtained for Thetis and Arete. It can be noted that the two results found for the mass of Vesta are very close to each other: the difference between them, $0.02310^{-10} M_{\odot}$, is less than $2 \%$, and approximately corresponds to the standard deviation on both results. This agreement enhances the confidence in the results. The weighting mean of the two values gives the final value $(1.306 \pm 0.014) 10^{-10} M_{\odot}$.

The correlation coefficient between the masses of Vesta and Parthenope, calculated from the global matrix $M$ (Sect. 3.1), is 0.41. This is a reasonable value that is explained by the fact that the mass of Vesta is determined using two test asteroids (the correlation coefficient is 0.60 if the data from Arete are omitted) and also by the fact that the major close encounter between Parthenope and Thetis and the close encounter between Vesta and Thetis are separated by 27 years (1968 versus 1995).

Along with the correlation coefficient, matrix $M$ provides the standard deviations of the masses determined, that take into account the correlation between both masses. These standard deviations are $0.01610^{-10} M_{\odot}$ for Vesta and $0.0710^{-12} M_{\odot}$ for Parthenope, which represents a slight increase from the values that do not take into account the correlation between the two masses.

It can be noted that our final result for the mass of Vesta is very close to the initial value assumed for this mass $\left(1.3510^{-10} M_{\odot}\right)$, and legitimate this choice. 
Table 5. New orbital elements of (17) Thetis, and standard deviations, at epoch JED $2450000.5=1995$ October 10.0 TT

\begin{tabular}{ccccccc}
\hline & $a(\mathrm{AU})$ & $e$ & $i\left(^{\circ}\right)$ & $\Omega\left(^{\circ}\right)$ & $\omega\left(^{\circ}\right)$ & $M\left(^{\circ}\right)$ \\
\hline & 2.469076562 & 0.13593536 & 5.585829 & 125.656706 & 136.130377 & 280.228176 \\
$\sigma$ & $2.710^{-9}$ & $1.810^{-8}$ & $1.610^{-6}$ & $1710^{-6}$ & $2210^{-6}$ & $1310^{-6}$ \\
\hline
\end{tabular}

Table 6. New orbital elements of (197) Arete, and standard deviations, at epoch JED 2450000.5=1995 October 10.0 TT

\begin{tabular}{ccccccc}
\hline & $a(\mathrm{AU})$ & $e$ & $i\left(^{\circ}\right)$ & $\Omega\left(^{\circ}\right)$ & $\omega\left(^{\circ}\right)$ & $M\left(^{\circ}\right)$ \\
\hline & 2.739420843 & 0.16302227 & 8.789733 & 81.879062 & 246.464868 & 168.770379 \\
$\sigma$ & $2110^{-9}$ & $6.210^{-8}$ & $7.310^{-6}$ & $3610^{-6}$ & $4910^{-6}$ & $3010^{-6}$ \\
\hline
\end{tabular}

We assumed the same a-priori value of the mass of Vesta in our previous papers, and in particular in Paper I for the first determination of the mass of Parthenope, when we took into account the perturbation of Vesta on the orbit of Thetis. In such mass determinations where gravitational perturbations from other asteroids influence the result found for the mass, it is necessary to use a good estimate of the masses of those perturbing asteroids.

It can also be noted that our final result for the mass of Vesta is in good agreement with most of other authors' (see Table 1), and in particular with Standish et al. (1998) and Michalak (2000), whose results were obtained using other test objets (planets and other test asteroids).

\subsection{Density of Vesta}

The accuracy of the mean density of Vesta is strongly dependent on the accuracy of its mean diameter, or its "effective" diameter (Bowell et al. 1989), that is, the diameter of the equal volume sphere. For asteroids having the shape of a triaxial ellipsoid like Vesta, the effective diameter $D_{\text {eff }}$ is given by

$D_{\text {eff }}=2(a b c)^{1 / 3}$

where $2 a, 2 b$ and $2 c$ are the ellipsoid axis (Müller \& Lagerros 1998). Determining an accurate effective diameter of Vesta is not the purpose of this paper, however we tried to find a good estimate from the literature. A summary of diameter determinations of Vesta is given in Table 7 .

The diameter used to calculate Vesta's mean density was determined by averaging the values given in Table 7 , and their scattering was used to calculate the standard deviation. Barnard's result and the IMPS value, both much smaller than other results, were rejected. The effective diameter found is $531 \pm 24 \mathrm{~km}$, which is logically very close to many results (TRIAD, Drummond et al. 1988; Müller \& Lagerros $1998, \ldots)$. It gives a mean density of $3.3 \pm 0.5 \mathrm{~g} / \mathrm{cm}^{3}$ for Vesta, to be compared to other authors' results (Table 8 ). This table summarizes the density determinations explicitely published, but it must be pointed out that most of the diameters listed in Table 7 give a mean density for Vesta between 3 and $4 \mathrm{~g} / \mathrm{cm}^{3}$ when associated with most of the mass determinations given in Table 1. This is a rather high value compared to other asteroids for which density determinations have been made,
Table 7. Summary of diameter determinations of Vesta. Part of these results were taken in Tedesco (1994). "3e" (resp. "2e") means that the shape was assumed to be a triaxial (resp. biaxial) ellipsoid

\begin{tabular}{lcl}
\hline Author & Technique & $\begin{array}{c}\text { Result } \\
(\mathrm{km})\end{array}$ \\
\hline Barnard (1900) & filar microm. & $385 \pm 50$ \\
Allen (1971) & radiometry & $570 \pm 10$ \\
TRIAD (1979) & polarimetry & $579 \pm 60$ \\
TRIAD (1979) & radiometry & $530 \pm 30$ \\
IRAS (1986) & radiometry & $501 \pm 24$ \\
Drummond et al. (1988) & speckle interf. & $525 \pm 13^{1}$ \\
Drummond \& Hege (1989) & speckle interf. & $520 \pm 25$ \\
Stamm (1989) & occultation & $561 \pm 3$ \\
Dunham (1991) & occultation & $520 \pm 10$ \\
IMPS (1992) & radiometry & $468 \pm 54$ \\
McCarthy et al. (1994) & IR speckle, 3e & $512 \pm 17^{1}$ \\
McCarthy et al. (1994) & IR speckle, 2e & $501 \pm 19^{1}$ \\
Thomas et al. (1997) & HST ${ }^{3}, 3 e$ & $516 \pm 24$ \\
Müller \& Lagerros (1998) & HST & 530.4 \\
Müller \& Lagerros (1998) & radiometry & $533.5 \pm 24$ \\
Müller \& Lagerros (1998) & radiom., STM & $537.6 \pm 29$ \\
\hline
\end{tabular}

${ }^{1}$ Calculated with Eq. (1).

${ }^{2}$ IRAS Minor Planet Survey.

${ }^{3}$ Hubble Space Telescope.

${ }^{4}$ Standard Thermal Model.

Table 8. Density determinations of Vesta

\begin{tabular}{lc}
\hline Author & Result $\left(\mathrm{g} / \mathrm{cm}^{3}\right)$ \\
\hline Schubart \& Matson (1979) & $3.3 \pm 1.5$ \\
Cellino et al. (1987) & $2.4 \pm 0.3$ \\
Drummond et al. (1988) & $3.62 \pm 0.35$ \\
Hilton (1999) & $4.3 \pm 0.3$ \\
\hline
\end{tabular}

since most of them show densities close to $2 \mathrm{~g} / \mathrm{cm}^{3}$ (Fig. 7). Our result therefore confirms a high density for Vesta, but we point out that this conclusion is strongly dependent on the effective diameter adopted for this asteroid. 


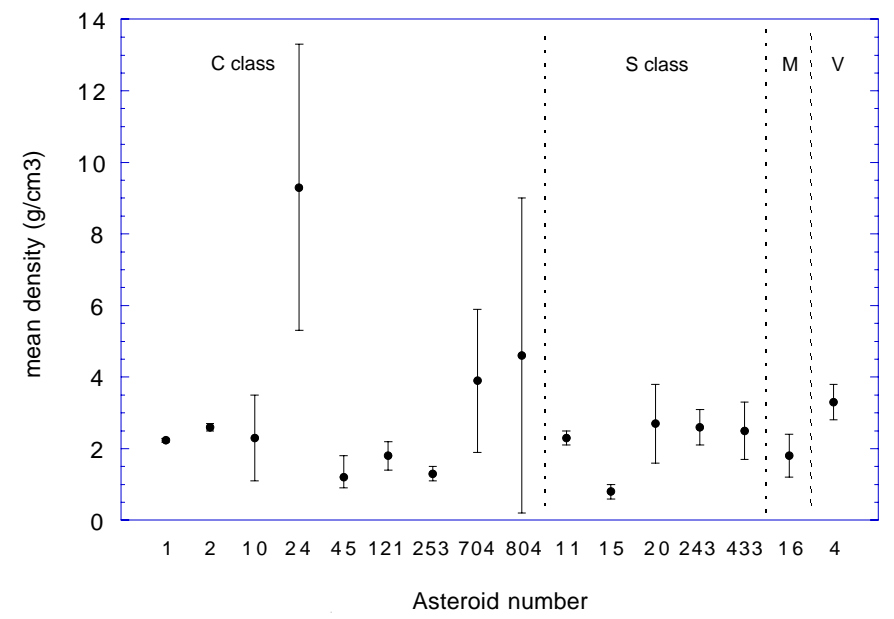

Fig. 7. Mean density of each of the 16 asteroids for which a mass has already been determined, sorted by class; references of the masses used (in brackets is given the asteroid number): [1] Viateau \& Rapaport (1998), [2] Standish et al. (1995), [4] this work, [10] Scholl et al. (1987), [11] this work, [15] Hilton (1997), [16] Viateau (2000), [20] Bange (1998), [24] López García et al. (1997), [45] Merline et al. (1999), [121] Viateau (2000), [243] Belton et al. (1995), [253] Yeomans et al. (1997), [433] Yeomans et al. (1999), [704 and 804] Landgraf (1992)

\section{Conclusion}

New values of mass and density were determined for two large asteroids, (4) Vesta and (11) Parthenope. The value found for the mass of Parthenope, $(2.56 \pm 0.07) 10^{-12} M_{\odot}$, confirmed and improved the result obtained in a previous paper. It gives a mean density of $2.3 \pm 0.2 \mathrm{~g} / \mathrm{cm}^{3}$, consistent with most of other S-class asteroids. The value obtained for the mass of Vesta is $(1.306 \pm 0.016) 10^{-10} M_{\odot}$ and gives a mean density of $3.3 \pm 0.5 \mathrm{~g} / \mathrm{cm}^{3}$, higher than most of other asteroids. The masses of Vesta and Parthenope are obtained with small standard deviations, but it is well known that the actual uncertainty of the results is higher due to observational bias and physical systematic effects, in particular gravitational effects of asteroids not yet taken into account in the physical model of the test asteroids used for the mass determination. A good point, however, is that our result for the mass of Vesta is in good agreement with recent results from authors who used other test objects to determine this mass, and also that Arete and Thetis, the two test asteroids used in this paper to determine the mass of Vesta, gave two very close results.

This is the first time asteroid (17) Thetis has been used to determine the mass of Vesta. Thetis is now the best target for this, and further observations of this asteroid, if made regularly, will help a lot to improve the knowledge of the mass of Vesta. A very good level of accuracy is needed for this mass, since Vesta is the second most massive asteroid and induces important gravitational perturbations on the orbits of many other solar system objects, including the planet Mars. Improvement of the mean densities of Vesta and Parthenope will be obtained only if the effective diameters of these asteroids can be known with a better accuracy, for example from star occultations. Observations of these phenomena are very useful, since our knowledge of sizes of asteroids is currently insufficient for most of the large asteroids.

Acknowledgements. The authors would like to thank the people from the meridian teams of Bordeaux and Valinhos (São Paulo) observatories, as well as Bill Owen, from the Jet Propulsion Laboratory, and Ronald C. Stone, from US Naval Observatory. All of these people contributed to the present work by making new and accurate observations of (17) Thetis. The authors also thank the referee, J. L. Hilton, for his helpful comments and suggestions.

\section{References}

Allen, D. A. 1971, In Physical Studies of Minor Planets, ed. T. Gehrels, NASA SP-267, 41

Bange, J. F. 1998, A\&A, 340, L1

Barnard, E. E. 1900, MNRAS, 60, 261

Batrakov, Y. V. 1994, Ephemerides of Minor Planets for 1995, Institute of Theoretical Astronomy, $\mathrm{S}^{\mathrm{t}}$ Petersburg

Belton, M. J. S., Chapman, C. R., Thomas, P. C., et al. 1995, Nature, 374, 785

Bowell, E., Hapke, B., Domingue, D., et al. 1989, in ed. R. P. Binzel, T. Gehrels, \& M. S. Matthews, Asteroids II (Univ. of Arizona Press, Tucson), 524

Bretagnon, P. 1982, A\&A, 114, 278

Bulirsh, R., \& Stoer, J. 1966, Num. Math., 8, 1

Cellino, A., Zappalà, V., Di Martino, M., et al. 1987, Icarus, 70,546

Drummond, J., Eckart, A., \& Hege, E. K. 1988, Icarus, 73, 1

Drummond, J. D., \& Hege, E. K. 1989, in ed. R. P. Binzel, T. Gehrels, \& M. S. Matthews, Asteroids II (Univ. of Arizona Press, Tucson), 171

Dunham, D. W. 1991, Occultation Newsletter, 5, 93

Eubanks, T. M. 1996, e-mails to "Friends of Mars Pathfinder"

Goffin, E. 1991, A\&A, 249, 563

Hertz, H. G. 1966, IAU Circ., 1983

Hertz, H. G. 1968, Science, 160, 299

Hilton, J. L., Seidelmann, P. K., \& Middour, J. 1996, AJ, 112, 2319

Hilton, J. L. 1997, AJ, 114, 402

Hilton, J. L. 1999, AJ, 117, 1077

Infrared Astronomical Satellite Asteroid and Comet Survey; Preprint Version No. 1, 1986, ed. D. L. Matson, JPL Internal Document NO. D-3698, Jet Propulsion Laboratory, Pasadena

IRAS Minor Planet Survey, 1992, ed. E. F. Tedesco, PL-TR92-2049, Phillips Laboratory, Hanscom Air Force Base, MA

Jefferys, W. H. 1963, AJ, 68, 111

Landgraf, W. 1992, in Proc. IAU Symp. 152, Chaos, resonance and collective dynamical phenomena in the Solar System, ed. S. Ferraz-Mello (Kluwer Academic Publishers, Dordrecht), 179

López García, A., Medvedev, Yu. D., \& Moraño Fernández, J. A. 1997, in Dynamics and Astrometry of Natural and Artificial Celestial Bodies, ed. I. M. Wytrzyszczak, J. H. Lieske, \& R. A. Feldman (Kluwer Academic Publishers, The Netherlands), 199

McCarthy, D. W., Freeman, J. D., \& Drummond, J. D. 1994, Icarus, 108, 285 
Merline, W. J., Close, L. M., Dumas, C., et al. 1999, Nature, 401, 565

Michalak, G. 2000, A\&A, 360, 363

Müller, T. G., \& Lagerros, J. S. V. 1998, A\&A, 338, 340

Ralston, A., \& Rabinowitz, P. 1978, A first course in numerical analysis (International Student Editions), 444

Scholl, H., Schmadel, L. D., \& Röser, S. 1987, A\&A, 179, 311

Schubart, J., \& Matson, D. L. 1979, in Asteroids, ed. T. Gehrels (University of Arizona Press, Tucson), 84

Sitarski, G. 1995, Acta Astron., 45, 673

Stamm, J. 1989, Reports of asteroidal appulses and occultations, Occultation Newsletter, 5, 327

Standish, E. M., \& Hellings, R. W. 1989, Icarus, 80, 326

Standish, E. M., Newhall, XX, Williams, J. G., \& Folkner, W. M. 1995, IOM 314.10-127, Jet Propulsion Laboratory, Pasadena, USA
Standish, E. M., Newhall, XX, Williams, J. G., et al. 1998, IOM 312.F-98-048, Jet Propulsion Laboratory, Pasadena, USA

Tedesco, E. F. 1994, in Asteroids, Comets, Meteors 1993, ed. A. Milani et al., 55

Thomas, P. C., Binzel, R. P., Gaffey, M. J., et al. 1997, Icarus, 128,88

Viateau, B. 2000, A\&A, 354, 725

Viateau, B., \& Rapaport, M. 1997, A\&A, 320, 652

Viateau, B., \& Rapaport, M. 1998, A\&A, 334, 729

Viateau, B., Réquième, Y., Le Campion, J. F., BenevidesSoares, P., Teixeira, R., et al. 1999, A\&AS, 134, 173

Yeomans, D. K., Barriot, J. P., Dunham, D. W., Farquhar, R. W., Giorgini, J. D., et al. 1997, Science, 278, 2106

Yeomans, D. K., Antreasian, P. G., Cheng, A., et al. 1999, Science, 285, 560 